\title{
HÁBITAT DEL VENADO COLA BLANCA EN LA SIERRA DE HUAUTLA, MORELOS, MÉXICO
}

\author{
Dante Alfredo Hernández Silva, ${ }^{1,2}$ Enrique Cortés DíAz, ${ }^{1}$ José Luis \\ Zaragoza Ramírez, ${ }^{1}$ Pedro Arturo Martínez Hernández, ${ }^{1}$ Giovany \\ Tonatiuh González Bonilla, ${ }^{3}$ Berta Rodríguez Castañeda ${ }^{1}$ y Dante Alfredo \\ Hernández SEdas 4 \\ ${ }^{1}$ Universidad Autónoma Chapingo. C.P. 56230. E-mail: ecodia@yahoo.com.mx, huextola2001@, \\ hotmail.com, pmartinezh@taurus.chapingo.mx, bertarc@correo.chapingo.mx \\ ${ }^{2}$ Autor corresponsal, E-mail: dal_silva@prodigy.net.mx \\ ${ }^{3}$ Agencia de Desarrollo Local Sierra de Huautla. Mor. C.P. 62923. E-mail: gtonatiuhg@yahoo.com.mx \\ ${ }^{4}$ C.B.T.a. No. 60. Cd. Hidalgo, Chiapas. A.P. 20. E-mail: dalsedas@hotmail.com
}

Hernández-Silva, D. A., E. Cortés-Díaz, J. L. Zaragoza-Ramírez, P. A. Martínez-Hernández, G. T. González-Bonilla, B. Rodríguez-Castañeda \& D. A. Hernández-Sedas. 2011. Hábitat del venado cola blanca, en la Sierra de Huautla, Morelos, México. Acta Zool. Mex. (n. s.), 27(1): 47-66.

RESUMEN. El estudio se realizó en las UMA's de Ajuchitlan-Santiopa, El Limón de Cuauchichinola y Pitzotlan, Morelos, México; de marzo a agosto de 2007, donde se evaluó el hábitat del venado cola blanca (Odocoileus virginianus mexicanus Gmelin, 1788). Para caracterizarlo se estimó el estrato arbóreo y arbustivo aplicando el método de puntos centrados en cuadrantes, del cual se estimó el diámetro de la copa, individuos vegetales en $100 \mathrm{~m}^{2}$, la accesibilidad de las plantas potencialmente consumidas por el venado, la altitud, la pendiente, valor de importancia de especies y familias vegetales e índices de riqueza (Margalef y Menhinick), equidad (Shannon y su inverso, Equidad o Uniformidad) y dominancia (Simpson y su inverso, Berger-Parker y su inverso). Se encontraron 72 especies vegetales, distribuidas en 29 familias, con dominancia de árboles de 68 y 32\% de arbustos. Las especies Euphorbia schlechtendalii, Lysiloma divaricata y Acacia cochliacantha, sobresalieron por su valor de importancia de especies, abundancia e importancia antropogénica. La familia Fabaceae fue la más representativa por el valor de importancia de familias. El análisis estadístico reveló que el área basal promedio fue similar entre UMA's y entre transectos $(p>0.05)$, así como la densidad de individuos vegetales en $100 \mathrm{~m}^{2}$ $(\bar{x}=23.44)$, la accesibilidad, pero las especies vegetales fueron altamente accesibles para el venado $(\mu=0.48 \mathrm{~m})$, la cobertura de la copa, con $\mu=8.89 \mathrm{~m}^{2}$ y la altitud; la pendiente fue diferente entre UMA's y entre transectos $(p<0.05)$. El hábitat del venado cola blanca en la Sierra de Huautla con mejores atributos, es aquel que presenta alta diversidad, abundancia y sin dominancia de especies vegetales y con mayor pendiente del terreno; enfocados principalmente en la disponibilidad de alimento y cobertura.

Palabras clave: Caracterización, riqueza, diversidad, venado cola blanca, Sierra de Huautla.

Hernández-Silva, D. A., E. Cortés-Díaz, J. L. Zaragoza-Ramírez, P. A. Martínez-Hernández, G. T. González-Bonilla, B. Rodríguez-Castañeda \& D. A. Hernández-Sedas. 2011. White-tailed deer habitat in the Huautla Sierra, Morelos, Mexico. Acta Zool. Mex. (n. s.), 27(1): 47-66.

Recibido: 03/03/2010; aceptado: 13/08/2010. 
ABSTRACT. The study was conducted in three certified units for environmental management (UMA), these were Ajuchitlan-Santiopa, El Limon de Cuauchichinola and Pitzotlan, Morelos, Mexico, it lasted from March to August, 2007, and consisted in the evaluation of white-tailed deer (Odocoileus virginianus mexicanus Gmelin, 1788) habitat. To define the habitat tree and shrub composition was determined by central point quadrants, measures were diameter of tree canopy, plant density in $100 \mathrm{~m}^{2}$, accessibility of potential plants that are ingested by deer, altitude, slope, species importance and botanical families and richness indexes (Margalef and Menhinick), equity (Shannon, Inverse Shannon, Evenness) and dominance indexes (Simpson, Inverse Simpson, Berger-Parker and Inverse Berger-Parker). A total of 72 species were found, 29 botanical families, tree and shrub dominances were 68 and 32\% respectively. Euphorbia schlechtendalii, Lysiloma divaricata and Acacia cochliacantha species showed the highest values in importance, abundance and even in anthropogenic use. The botanical family Fabaceae was the most wide spread and with the highest importance value among botanical families. From statistical analysis mean basal wasn't different between UMA's and transects $(p>0.05)$ as well as plant density in $100 \mathrm{~m}^{2}(\bar{x}=23.44)$, the accessibility with a mean value of $0.48 \mathrm{~m}$ which was highly accessible to deer, areal tree cover of $8.89 \mathrm{~m}^{2}$ and the altitude; the slopes was different between UMA's and transects $(p<0.05)$. The white-tailed deer habitat of the Sierra de Huautla with the best attributes is the one that shows high richness, abundance and no dominance of plant species and high slopes on the field; focus mainly on food availability and coverage.

Key words: Characterization, richness, diversity, white-tailed deer, Sierra de Huautla.

\section{INTRODUCCIÓN}

El hábitat determina la presencia de una especie animal en un lugar en específico (Trefethen 1964), con ello se crea la importancia de conocer las características del hábitat de especies de fauna silvestre de interés cinegético, para asegurar la permanencia de poblaciones y lograr un aprovechamiento racional. Las evaluaciones de hábitats implican conocer las características que presenta el área que habita una determinada especie, comprende a una serie de componentes que influyen de manera directa sobre el número y distribución de los animales (Gallina 1998). En el caso de la conservación y aprovechamiento sustentable del venado cola blanca, el estudio de hábitat es prioritario para el manejo y debe incluir el conocimiento de las necesidades básicas de la especie (Delfín-Alfonso \& Gallina 2007).

El hábitat de alta calidad, se define como las áreas que presentan las condiciones necesarias para incrementar la adaptación de los individuos de una población durante períodos prolongados de tiempo (Gallina, 1998); para el venado cola blanca, debe integrar cuatro elementos básicos: alimento y agua disponible; espacio como áreas de apareamiento, nacimiento y crianza; cobertura de protección contra el clima, depredadores, de traslado, pernoctación y descanso durante el día (Gallina 1998, Bello et al. 2004, Lyons \& Ginnet 2001, Ayala \& Quintero 2003, Fulbright \& Ortega 2007, Hernández 2008). La riqueza de especies es de gran valor biológico para el venado cola blanca, Soto (2002) y Velázquez et al. (2003) mencionan que la riqueza de especies vegetales es factor importante para la selección del hábitat del venado cola blanca, basado principalmente en la diversidad vegetal que esta especie pueda aprovechar como forraje en un área determinada (Villarreal et al. 2007). 
En la parte noreste de la Reserva de la Biosfera Sierra de Huautla (REBIOSH), se localizan los ejidos Ajuchitlan-Santiopa, El Limón de Cuauchichinola y Pitzotlan, donde se establecieron Unidades de Manejo para la Conservación y Aprovechamiento de la Vida Silvestre (UMA) extensivas, cuyos objetivos son conservación y aprovechamiento sustentable del venado cola blanca y su hábitat, de manera sistemática.

Con base en estudios de densidad de población del venado cola blanca en las UMA's de la Sierra de Huautla (Cruz 2004, González 2005, González 2006, Hernández 2008) se han obtenido permisos para realizar aprovechamiento cinegético y de subsistencia, además de contribuir a realizar acciones mediatas e inmediatas para implementar estrategias de manejo, enfocadas a la conservación y aprovechamiento extractivo racional de la especie. No existen estudios de hábitat en la región que ocupan las UMA's, por lo tanto, este trabajo planteó el objetivo de caracterizar el hábitat del venado cola blanca mexicanus en UMA's de la Sierra de Huautla.

\section{MATERIALES Y MÉTODOS}

El área de estudio comprende a tres UMA's extensivas en el estado de Morelos, México: UMA Cinegético El Metate (2,783 ha), ejido Ajuchitlan municipio de Tlaquiltenango, UMA Cinegético El Limón de Cuauchichinola (4,256 ha), ejido El Limón de Cuauchichinola municipio de Tepalcingo y UMA Venado cola blanca y animales silvestres de Pitzotlan (1,694 ha), ejido de Pitzotlan municipio de Tepalcingo (Figura 1). En lo sucesivo, se manejará los nombres de las UMA's refiriéndose al nombre del ejido; como UMA Ajuchitlan, UMA El Limón y UMA Pitzotlan.

El clima es cálido subhúmedo con lluvias en verano y presencia de canícula, temperatura media anual de $23.26^{\circ} \mathrm{C}$. La temperatura más alta ocurre en abril o mayo. La precipitación anual es de 861.83 mm (García 1981, SMN 1976-2000).

En la región existen dos épocas bien definidas durante el año, la sequía abarca de mediados de noviembre a mediados de abril y la época de lluvias, de mediados de mayo a mediados de noviembre (Hernández 2008). La vegetación dominante es la selva baja caducifolia (SBC), característica de la Sierra de Huautla, misma que presenta diferencias muy marcadas por su composición florística con el resto de las SBC del país (Arías et al. 2002). Además de existir gran diversidad de fauna (Sánchez \& Romero 1995; Arías et al. 2002; Dorado et al. 2002; Ramírez \& Ramírez 2002; Castro \& Bustos 2003; CONANP 2005; Rodríguez \& Woolley 2005; Castro et al. 2006; Ávalos 2007).

Se caracterizó el hábitat del venado cola blanca, utilizando el método de puntos centrados en cuadrantes (Muller-Dombois \& Ellenberg 1974; Cox, 1978) sobre transectos de $1,000 \mathrm{~m}$ de longitud perpendiculares a la curvas de nivel (no se realizaron a lo largo de las barrancas, sobre caminos o veredas), el área de estudio se dividió en bloques de un kilómetro cuadrado, los transectos se seleccionaron de manera aleatoria de acuerdo a lo accidentado del terreno y fueron nombrados dependiendo del paraje o 


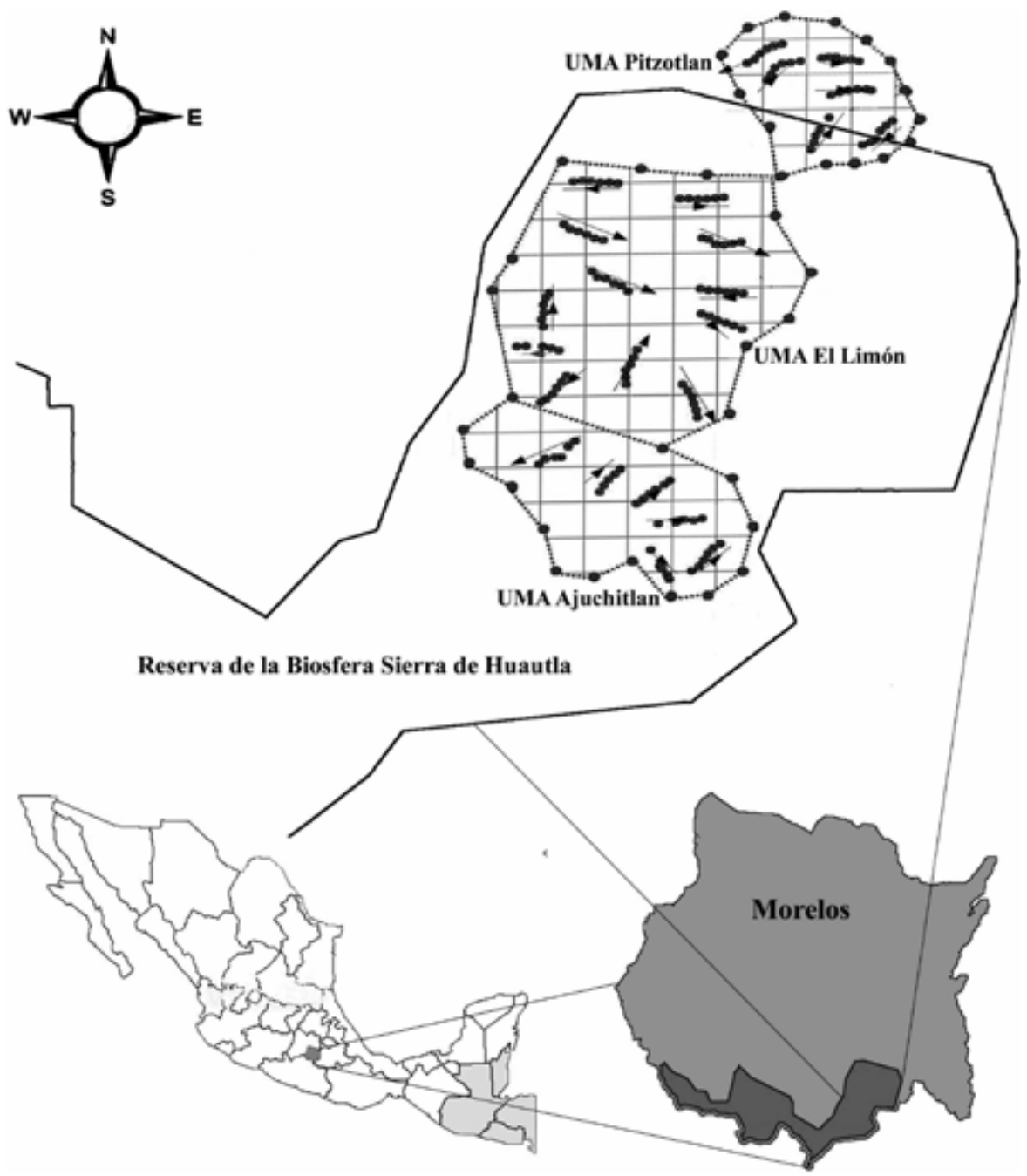

Figura 1. Macro y microlocalización de las UMA's en estudio (Reserva de la Biosfera Sierra de Huautla).

al cerro donde se localizaban cada uno de ellos, el muestreo fue dirigido a arbustos y árboles principalmente. A lo largo del transecto, se estableció un punto de muestreo a cada $100 \mathrm{~m}$ (once en total para cada transecto), donde se midió la distancia al punto central, la especie vegetal, el diámetro de árboles y arbustos a una altura de 60 y 30 $\mathrm{cm}$ respectivamente, la cobertura de la copa de árboles y arbusto, mediante el método del diámetro de la copa (Muller-Dombois \& Ellenberg 1974), la accesibilidad de cada especie arbórea y arbustiva (Hernández 2008) entendida como la biomasa de la 
planta en la cual el venado cola blanca puede realizar el forrajeo (Mandujano 1994), la altitud, la pendiente del terreno (plancheta dendométrica) y la presencia de agua para cada transecto. En cada UMA se estimó el área basal de cada especie vegetal y la densidad total de plantas (por $100 \mathrm{~m}^{2}$ ) obtenida por la división del área común por planta dentro de la unidad de área (Cox 1978). Se identificaron las especies de árboles y arbustos in situ por su nombre común, comparándolos con las especies identificadas por González \& López (2008) en la UMA El Limón, así mismo la preferencia de especies vegetales que tiene el venado cola blanca mediante observaciones directas y con ayuda de los pobladores humanos.

Con la finalidad de documentar la diversidad vegetal, se calcularon índices de diversidad en el hábitat de las UMA's en estudio. Se determinó la riqueza de especies (S) (Magurran 1989), el índice de valor de importancia (IVI) de especies (Lamprecht 1990); índice de valor de importancia de familias (IVIF) (Mori et al. 1983), los índices de diversidad: índice de Margalef $\left(D_{M g}\right)$, índice de Shannon $\left(H_{S}\right)$ y su Uniformidad $(E)$, índice de Simpson $(D)$ y el índice Berger-Parker $\left(D_{B P}\right)$, de acuerdo con Magurran (1989).

Los índices de diversidad vegetal y las características del hábitat área basal, distancia promedio, cobertura de copa, individuos por $100 \mathrm{~m}^{2}$, accesibilidad, pendiente y altitud fueron analizadas dentro de cada UMA y entre UMA's, mediante el procedimiento de modelos mixtos del programa $\mathrm{SAS}^{\circledR}$, usando la prueba $t$ de comparación de medias.

\section{RESULTADOS}

Se encontraron un total de 72 especies de árboles y arbustos en las tres UMA's; las especies arbóreas dominaron sobre las arbustivas (68vs 32\%). El 99\% de las especies encontradas son caducifolias, el resto pertenecen a especies columnares y candelubriformes (Apéndice 1). En total se encontraron 29 familias; siendo Fabaceae la dominante con $32 \%$ de los individuos totales, seguida de Burseraceae, Cactaceae, Euphorbiaceae, Convolvulaceae, 18 familias con una especie y dos familias no fueron identificadas (Figura 2). La UMA El Limón presentó la mayor riqueza de especies ( $p$ $<0.05$ ), superior en $75 \%$ del total de especies y superior en 21 y $15 \%$ con las UMA's Ajuchitlan y Pitzotlan respectivamente (Cuadro 1).

El hábitat del venado cola blanca en las UMA's presentó área basal de $1.24 \mathrm{~m}$, la distancia promedio fue de $2.19 \mathrm{~m}$, en cuanto a la accesibilidad $(\bar{x}=0.48 \mathrm{~m})$; la UMA El Limón presentó 60.34 y $68.63 \%$ menor accesibilidad que Ajuchitlan y Pitzotlan; se estimaron 24 individuos en $100 \mathrm{~m}^{2}$ en las tres UMA's, donde también la UMA El Limón fue mayor $\left(27\right.$ ind $\left.100 \mathrm{~m}^{-2}\right)$; la cobertura de la copa $\left(\bar{x}=8.89 \mathrm{~m}^{2}\right)$ fue mayor en la UMA Ajuchitlan $\left(11.02 \mathrm{~m}^{2}\right)$; se presentaron pendientes promedio por encima del $28 \%\left(16.05^{\circ}\right)$, la UMA El Limón presentó pendientes más pronunciadas $\left(\bar{x}=19.16^{\circ}\right.$ 


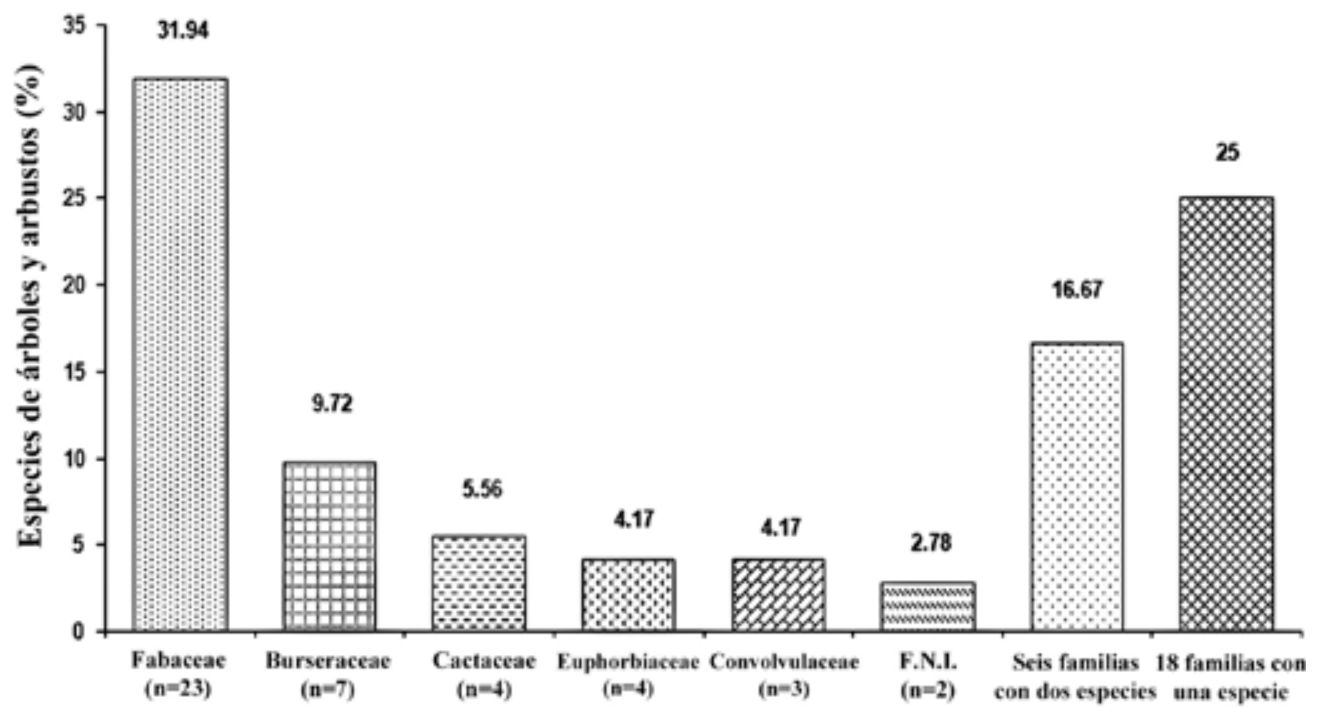

Familias vegetales

Figura 2. Porcentaje de las principales familias vegetales encontradas en las UMA's en estudio. Claves usadas: $\mathrm{n}$ (número de individuos vegetales), F.N.I. (familia no identificada).

o $34.75 \%)$ y la UMA Pitzotlan presentó las menores $\left(\bar{x}=13.11^{\circ}\right.$ o $\left.23.29 \%\right)$; la mayor altitud se presentó en la UMA Ajuchitlan (1772 msnm) y la menor en la UMA Pitzotlan (1158 msnm) (Cuadro 1).

De acuerdo con los índices de diversidad vegetal, la UMA El Limón presentó mayor diversidad de especies arbóreas y arbustivas, lo cual corresponde a que en esta UMA se ha trabajado conjuntamente desde hace aproximadamente 20 años, por lo tanto, se ha logrado un ambiente de conservación y aprovechamiento de los recursos naturales. En la parte ecológica, los pobladores humanos de la UMA han observado un crecimiento en la población del venado cola blanca, resultado de la vigilancia participativa, cacería únicamente en la temporada permitida y conservación del hábitat (Hernández 2008).

La figura 3, muestra las especies con más éxito ecológico, por su alta dominancia, densidad y frecuencia relativa. En la UMA Ajuchitlan fueron Euphorbia schlechtendalii, Mimosa benthami y Lysiloma divaricata; las tres especies sumaron el 37.29\% del total del índice de valor de importancia de especies (IVI) de la UMA. Las especies más exitosas en la UMA El Limón fueron Euphorbia schlechtendalii, Lysiloma divaricata y Ficus cotinifolia (Figura 3), éstas representaron un $32.77 \%$ del total del IVI de la UMA. La UMA Pitzotlan presentó como especies más exitosas Euphorbia schlechtendalii, Cordia curassavica y Lysiloma divaricata, las cuales sumaron el 
Cuadro 1. Índices de diversidad y características del hábitat del venado cola blanca para las UMA's del estudio.

\begin{tabular}{|c|c|c|c|c|}
\hline UMA & Ajuchitlan & El Limón & Pitzotlan & Promedio \\
\hline $\mathrm{S}$ & 43 & 54 & 39 & 72 \\
\hline $\mathrm{n}$ & 24 & 48 & 24 & 32 \\
\hline Venados km $\mathrm{km}^{-2}(1)$ & $13.52 \pm 0.88$ & $12.78 \pm 0.65$ & $9.73 \pm 0.75$ & 12.01 \\
\hline $\mathrm{D}_{\mathrm{Mn}}$ & 2.54 & 3.02 & 2.04 & 2.53 \\
\hline $\mathrm{D}_{\mathrm{Mg}}$ & 3.92 & 5.36 & 3.13 & 4.14 \\
\hline$H^{\prime}$ & 2.26 & 2.67 & 1.90 & 2.28 \\
\hline $\mathrm{E}$ & 0.90 & 0.89 & 0.83 & 0.87 \\
\hline $\mathrm{D}$ & 0.097 & 0.077 & 0.174 & 0.12 \\
\hline $1 / \mathrm{D}$ & 11.09 & 14.01 & 6.56 & 10.55 \\
\hline $\mathrm{D}_{\mathrm{BP}}$ & 0.26 & 0.21 & 0.34 & 0.27 \\
\hline $1 / \mathrm{D}_{\mathrm{BP}}$ & 4.10 & 5.02 & 4.80 & 4.64 \\
\hline $\mathrm{N} \max (\mathrm{n})$ & $\begin{array}{c}\text { Euphorbia } \\
\text { schlechtendalii }\end{array}$ & $\begin{array}{c}\text { Euphorbia } \\
\text { schlechtendalii }\end{array}$ & $\begin{array}{c}\text { Euphorbia } \\
\text { schlechtendalii }\end{array}$ & $\begin{array}{c}\text { Euphorbia } \\
\text { schlechtendalii }\end{array}$ \\
\hline A.B. $\left(\mathrm{m}^{2}\right)$ & 1.33 & 1.17 & 1.21 & 1.24 \\
\hline $\begin{array}{c}\text { Distancia } \\
\text { promedio }(\mathrm{m})\end{array}$ & 2.48 & 1.95 & 2.12 & 2.19 \\
\hline Accesibilidad (m) & 0.58 & 0.35 & 0.51 & 0.48 \\
\hline Ind $100 \mathrm{~m}^{-2}$ & 18.70 & 26.97 & 24.64 & 23.44 \\
\hline $\begin{array}{l}\text { Cob de la copa } \\
\qquad\left(\mathrm{m}^{2}\right)\end{array}$ & 11.02 & 8.84 & 6.81 & 8.89 \\
\hline Pendiente $\left({ }^{\circ}\right)$ & 15.88 & 19.16 & 13.11 & 16.05 \\
\hline Altitud (msnm) & 1407.99 & 1300.96 & 1428.84 & 1379.26 \\
\hline
\end{tabular}

$\mathrm{S}$ (Riqueza de especies), $\mathrm{n}$ (Total de especies muestreadas por transecto), $\mathrm{D}_{\mathrm{Mn}}$ (Índice Menhinick), $\mathrm{D}_{\mathrm{Mg}}$ (Índice de Margalef), H' (Índice de Shannon), E (Igualdad o equidad), D (Índice de Simpson), ID (Inverso del Índice de Simpson), $\mathrm{D}_{\mathrm{BP}}$ (Índice de Berger-Parker), Nmax (Especie más abundante), A.B. (Área basal), Ind $100 \mathrm{~m}^{-2}$ (Número de individuos vegetales por cada $100 \mathrm{~m}^{2}$ ), Cob de la copa (Cobertura de la copa).

${ }^{1}$ Hernández (2008).

$42.29 \%$ del total del IVI de la UMA (Figura 3). Del total de familias de plantas que se encontraron en la región que ocupan las tres UMA's, 21 de ellas se presentaron en la UMA Ajuchitlan; Fabaceae presentó un índice de valor de importancia de Familias (IVIF) de $40.36 \%$, que sumada a las familias Euphorbiaceae y Burseraceae comprenden el 69.42\% del IVIF total (Cuadro 2). En la UMA El Limón, se encontraron 26 familias; Fabaceae presentó 36.16\% del IVIF del total, en segundo lugar, se ubica la familia Euphorbiaceae y en tercero la familia Burseraceae, las tres familias sumaron 58.07\% del IVIF del total (Cuadro 2). Se encontraron 17 familias en la UMA Pit- 

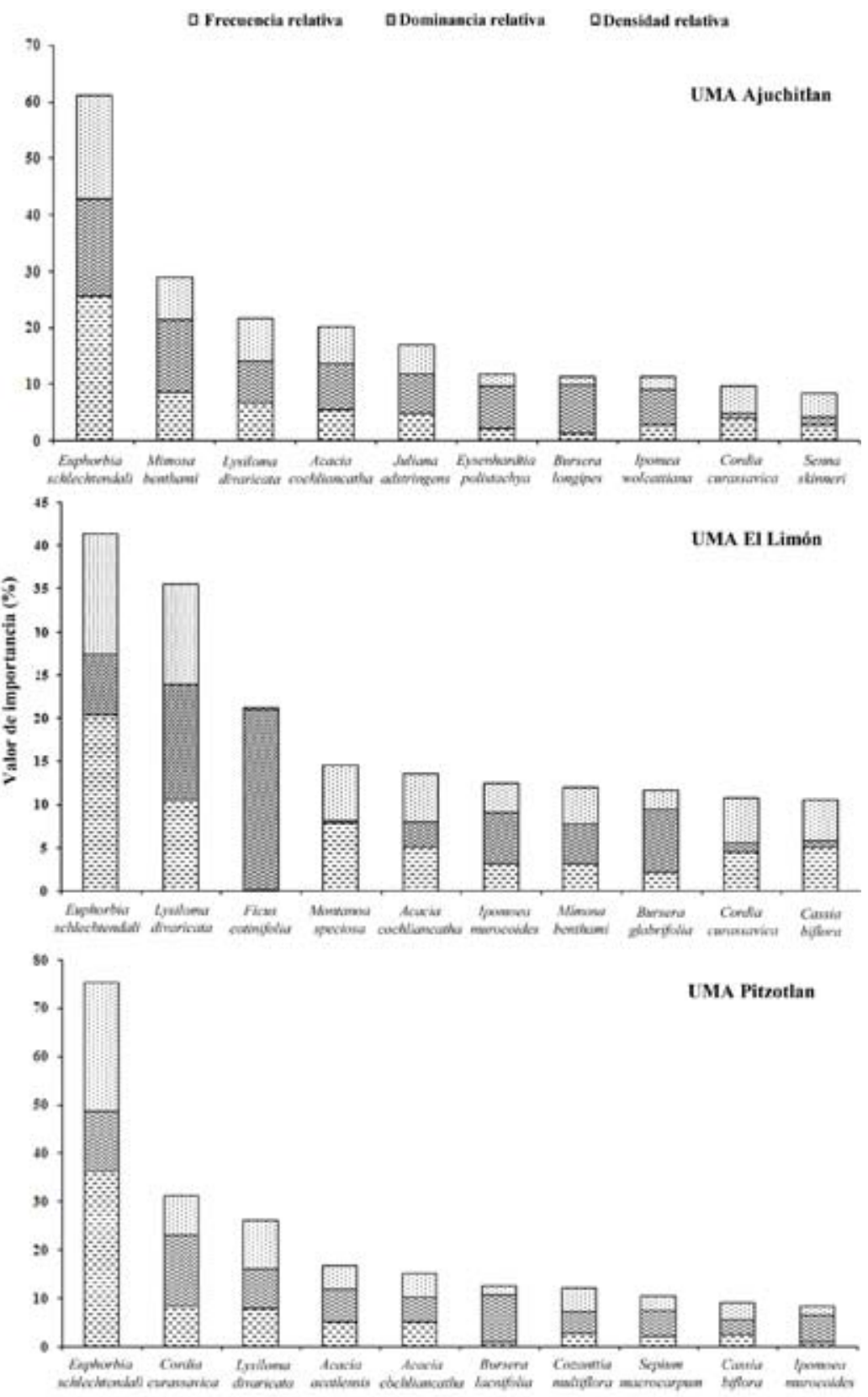

Especies vegetales

Figura 3. Valor de importancia de las 10 principales especies en las UMA's en estudio. 
Cuadro 2. Índice de valor de importancia de las principales familias encontradas en las UMA's del estudio.

\begin{tabular}{clrrrrrrrrr}
\hline UMA & Familia & $\begin{array}{c}\text { Ind } \\
100 \mathrm{~m}^{2}\end{array}$ & $\begin{array}{c}\text { A.B. } \\
\left(\mathrm{m}^{2}\right)\end{array}$ & \multicolumn{1}{c}{$\mathrm{A}$} & $\begin{array}{r}\text { No. } \\
\mathrm{Sp}\end{array}$ & $\begin{array}{c}\text { DIR } \\
(\%)\end{array}$ & $\begin{array}{c}\text { FR } \\
(\%)\end{array}$ & $\begin{array}{r}\text { DO } \\
(\%)\end{array}$ & VIF & IVIF \\
\hline Ajuchitlann & Fabaceae & 1.08 & 1.99 & 92 & 14 & 34.85 & 37.97 & 48.26 & 121.08 & 40.36 \\
& Euphorbiaceae & 11.17 & 0.85 & 68 & 1 & 25.76 & 18.18 & 17.10 & 61.03 & 20.34 \\
& Burseraceae & 0.78 & 2.26 & 19 & 4 & 7.20 & 8.02 & 10.93 & 26.15 & 8.72 \\
& Julianaceae & 0.16 & 0.08 & 13 & 1 & 4.92 & 5.35 & 6.93 & 17.20 & 5.73 \\
& Convolvulaceae & 0.99 & 1.68 & 12 & 2 & 4.55 & 4.28 & 7.13 & 15.95 & 5.32 \\
El Limón & Fabaceae & 1.34 & 1.44 & 198 & 17 & 37.50 & 40.11 & 30.88 & 108.49 & 36.16 \\
& Euphorbiaceae & 3.80 & 0.57 & 113 & 3 & 21.40 & 15.04 & 7.63 & 44.07 & 14.69 \\
& Burseraceae & 0.63 & 2.14 & 25 & 4 & 4.73 & 5.85 & 11.06 & 21.64 & 7.21 \\
& Moraceae & 0.10 & 123.51 & 1 & 1 & 0.19 & 0.28 & 20.82 & 21.29 & 7.10 \\
& Boraginaceae & 2.27 & 0.22 & 45 & 2 & 8.52 & 9.47 & 1.66 & 19.65 & 6.55 \\
& Fabaceae & 0.56 & 0.99 & 84 & 13 & 32.31 & 38.27 & 30.19 & 100.77 & 33.59 \\
& Euphorbiaceae & 2.43 & 0.91 & 112 & 4 & 43.08 & 32.10 & 18.40 & 93.58 & 31.19 \\
& Boraginaceae & 1.91 & 2.24 & 22 & 1 & 8.46 & 8.02 & 14.84 & 31.33 & 10.44 \\
& Burseraceae & 0.22 & 4.68 & 11 & 5 & 4.23 & 6.79 & 14.25 & 25.27 & 8.42 \\
& Convolvulaceae & 0.17 & 4.06 & 4 & 2 & 1.54 & 2.47 & 6.20 & 10.21 & 3.40 \\
\hline
\end{tabular}

Ind $100 \mathrm{~m}^{2}$ (Individuos vegetales en $100 \mathrm{~m}^{2}$ ), A.B. $\left(\mathrm{m}^{2}\right)$ (Área Basal), A (Abundancia de individuos de la familia), No. Sp (Número de especies que conforman la familia), DIR (Diversidad relativa), FR (Frecuencia relativa), DO (Dominancia relativa), VIF (Valor de importancia de familias), IVIF (Índice de valor de importancia de familias).

zotlan, de las cuales Fabaceae presentó el mayor IVIF con 33.59\%, seguido por la familia Euphorbiaceae y la familia Boraginacea. Las tres familias sumaron un IVIF de $77.23 \%$ del total calculado para el grupo de familias que se encontraron presentes en esta UMA (Cuadro 2).

\section{DISCUSIÓN}

La composición florística en las UMA's fue diferente; la de El Limón presentó 11 y 15 especies vegetales más que las UMA's de Ajuchitlan y Pitzotlan, respectivamente (Apéndice 1). Lo anterior se debe, a que por acuerdo comunitario se permite el pastoreo de bovinos y équidos; que implica la diseminación de las semillas de las plantas a través del proceso digestivo de los animales apacentadores. Del total de las especies de las tres UMA's solo 21 de ellas (29\%) fueron comunes, de este grupo sobresalen tres por su abundancia, importancia antropogénica y fuente de alimento para la fauna silvestre, Euphorbia schlechtendalii, es una fuente importante de alimento para el venado que rebrota en la época seca; Acacia cochliacantha y Lysiloma divaricata proporcionan rebrote, flor y vaina para el venado. Las especies Euphorbia schlechtendalii y Lysiloma divaricata comparten las características de ser dominantes en la comunidad vegetal en la región y en general para la SBC de México. Trejo (1998) encontró que Lysiloma divaricata contiene el valor de importancia más alto 
en la UMA El Limón, mostrando importancia a nivel antropogénico para el ganado y la fauna silvestre.

Desde el punto de vista ecológico, la dominancia de árboles se debe a que durante las campañas de muestreo, las herbáceas se encontraban a la mitad del período de crecimiento (González \& López 2008) por lo que no se identificó esta categoría de forma biológica de plantas.

Trejo (2005) menciona que una de las características de la SBC de México es la dominancia de especies vegetales caducifolias lo cual estuvo presente en las UMA's del estudio (Apéndice 1), además, que son fisonómicamente dominadas por árboles que se ramifican a baja altura y de copas extendidas, el estrato arbustivo es muy denso, de tal manera que en algunos sitios forman una maraña que dificulta el paso, además de presentar cactáceas columnares y candelubriformes, como variantes de la selva, que contrastando con estudios realizados por Durán et al. (2006) y González \& López (2008), quienes encontraron una dominancia de arbustos y herbáceas en SBC. Lo anterior es favorable porque una menor disposición de arbustos es perjudicial para un ecosistema, al disminuir la materia orgánica del suelo y crear condiciones no favorables para el crecimiento arbustos y herbáceas (Hernández 2008). Si faltan los arbustos se acelera el proceso de erosión, desertificación, disminución de alimento y cobertura para animales silvestres y domésticos y disminuyen los beneficios antropogénicos (Gutiérrez \& Squeo 2004).

Durante el estudio en las UMA's hubieron avistamientos de rastros de coyote ( $\mathrm{Ca}$ nis latrans), puma (Puma concolor), zorra gris (Urocyon cinereoargenteus) y tigrillo (Leopardos wiedii) además se observaron aves rapaces y de carroña.

La familia Fabaceae fue la más dominante en las tres UMA's, mismo resultados reportan Trejo (1998), Nogueda (2007) y González \& López (2008) para el caso de El Limón. La importancia de la familia Fabaceae esta refleja por contribuir con un $37.5 \%$ en la dieta y con un $45.22 \%$ del contenido de materia seca que requiere el venado cola blanca que habita en la Mixteca Poblana (Villarreal et al. 2007).

En cuanto a las características del hábitat, el área basal expresa la dominancia de los árboles y arbustos, el promedio para las tres UMA's y por transecto fue similar $(p>0.05)$ (Cuadro 1) y es menor comparado con las SBC de México $\left(5.6 \mathrm{~m}^{2}\right)$ reportado por Trejo (2005). La distancia promedio de árboles y arbustos fue semejante $(p>0.05)$ entre UMA's y entre transectos; con la distancia promedio de los transectos de cada UMA se calculó la densidad total de plantas, la cual fue mayor en la UMA El Limón con 525.72 individuos en los $1000 \mathrm{~m}$ de longitud de cada transecto, superando por 364 plantas a la UMA Ajuchitlan y con 304 plantas a la UMA Pitzotlan. Estos valores influyeron en la cobertura de protección y en la presencia del venado cola blanca en los transectos donde la vegetación ofreció mayor protección (Hernández 2008).

La accesibilidad, fue similar $(p>0.05)$ en las tres UMA's y entre transectos, El Limón presentó 60.34 y $68.63 \%$ menor accesibilidad que las de Ajuchitlan y Pitzot- 
lan, respectivamente (Cuadro 1), sin embargo, estos valores de árboles y arbustos son altamente accesibles al estar dentro del rango de 0 a $1.5 \mathrm{~m}$, el cual refleja que existe gran disponibilidad del alimento para el venado cola blanca. Del total de especies vegetales, el $72 \%$ son consumidas por el venado cola blanca y el resto no son preferidas (Apéndice 1). Los árboles y arbustos favorecen la disponibilidad de alimento, principalmente en el sotobosque (estrato arbustivo y herbáceo) porque conforman un 30 a $50 \%$ de las partes accesibles para el venado (hojas y retoños); el resto de las partes consumibles (semillas, flor y frutos) son accesibles hasta que se desprenden de los árboles y arbustos.

En el hábitat de la UMA El Limón se estimaron 53.12 ind $100 \mathrm{~m}^{-2}$. De acuerdo con Hernández (2008) en el transecto donde se estimó este valor, también se presentó el mayor número de venados de todas las UMA's $\left(21.3\right.$ ind $\left.\mathrm{km}^{-2}\right)$. Lo anterior concuerda con Ortíz et al. (2005) quienes encontraron la mayor densidad del venado cola blanca en sitios donde la cobertura y volumen de los arbustos y la pendiente fueron mayores que los demás sitios, bajo condiciones de la sierra norte de Oaxaca. Las especies más importantes para brindar esta cobertura de protección en las tres UMA's fueron Lonchocarpus eriophyllus, Ceiba parvifolia, Vitex pyramidata, Bursera longipes, Eysenhardtia polystachya, Ficus cotinifolia, Leucaena collinsii, Malpigia mexicana, Guazuma ulmifolia, Bursera lacifolia y Trichilia hirta; sin embargo, el resto de los individuos vegetales también brindan cobertura para la fauna silvestre. No existió diferencia $(p>0.05)$ en la densidad de árboles y arbustos por especie para cada UMA (Cuadro 1) y entre transectos. La importancia de la cobertura de protección de árboles y arbustos, así como la presencia de los mismos en el hábitat del venado, es para esconderse y escapar de los depredadores y protegerse de los fenómenos climáticos (Starker 2000; Lyons \& Ginnet 2001); además, los venados machos prefieren sitios (echaderos) con densa vegetación herbácea y/o arbustiva, mientras que las hembras buscan sitios con mayor cobertura de protección principalmente en la época de nacimientos de cervatos para permanecer escondidos (Gallina et al. 2010). Por lo tanto, los arbustos son componente principal del hábitat del venado cola blanca (Montemayor et al. 1991; Ramírez et al. 1997; Gallina et al. 2010). Un efecto de la falta de cobertura de protección por árboles y arbustos de alguna manera favorece a los cazadores con o sin autorización, al incrementar la visibilidad dentro del hábitat.

La proporción del suelo cubierta por la copa de los árboles y arbustos, fue similar $(p>0.05)$ entre UMA's (Cuadro 1) y entre transectos. Corona (2003) encontró valores diferentes de cobertura de copa de árboles y similares en la copa de arbustos, en estudio realizado en la UMA El Limón; tal diferencia puede deberse a que los muestreos incluyeron barrancas. Este tipo de cobertura es principalmente térmica al ofrecer sombra y refugio para fenómenos climáticos, López-Téllez et al. (2007) asoció la densidad del venado cola blanca en la Mixteca Poblana con la cobertura térmica 
y Gallina et al. (2010) la relacionó con las preferencias de sitios por los machos de esta especie.

La pendiente de los transectos de las tres UMA's fue diferente $(p<0.05)$, en la UMA Pitzotlan donde se estimó menor cantidad de venados por kilometro cuadrado $(9.73 \pm 0.75)$, presentó menores pendientes (Cuadro 1), mientras que la UMA Ajuchitlan y El Limón, donde se presentaron los transectos más accidentados $\left(\bar{x}=27^{\circ}\right)$ se estimó mayor cantidad de venados por kilometro cuadrado. Estos datos concuerdan con Corona (2003) al estimar mayor presencia de venados en transectos más accidentados y con mayor sinuosidad ( 38 a $44^{\circ}$ de pendiente). De acuerdo con Villarreal (2006) no existe competencia con el ganado bovino cuando se evita el sobrepastoreo y haya espacio suficiente para ambas especies. Al liberar el ganado bovino en el agostadero en las UMA's en estudio la disponibilidad de forrajes es mínima por efecto de la sequía, con ello se observa el consumo arbustos y herbáceas comunes en la dieta de ambas especies. En las áreas donde se observó lo anterior durante los muestreos y por comunicación personal por parte de los pobladores humanos de las UMA's, existe sobrepastoreo. Durante la época de sequía las dietas de ambas especies indican un pequeña sobreposición (Gallina et al. 1981), porque en la dieta del venado cola blanca el consumo de herbáceas es ocasional, mientras que en el ganado bovino es el componente principal (Drawe \& Box 1968, Everitt \& Drawe 1974, Gallina 1993). Gallina et al. (1981) mencionaron que el consumo de herbáceas por el venado aumenta durante la época de lluvias; sin embargo, los bovinos son de mayor tamaño y con ello consumen más forraje por día que un venado, entonces su consumo absoluto de herbáceas y gramíneas puede ser igual o mayor al de los venados aún cuando éstas constituyan sólo un porcentaje pequeño en la dieta (Fulbright y Ortega 2007), entonces el ganado bovino si compite por alimento con el venado cola blanca (McMahan y Ramsey 1965).

La región que ocupan las UMA's se caracteriza por ser un terreno accidentado, caracterizado por cerros de grandes altitudes; de acuerdo con López-Telléz et al. (2007) la densidad del venado cola blanca también se puede asociar a la altitud, con esta acción el venado logra evitar al ganado bovino buscando altitudes mayores, que son poco accesibles para los mismos (Hernández 2008), durante los muestreos se encontraron lugares donde no existieron rastros ni avistamientos de ganado bovino, pero sí de venados, principalmente en la cima de los cerros más altos.

De acuerdo con Corral et al. (2002) el valor obtenido para el índice de Menhinick, se sobreestimó, porque recomienda implementar transectos muy grandes o mayores a 1,200 m para calcular la diversidad. Para el índice de Margalef, la UMA Ajuchitlan presentó el valor más alto $(\mathrm{DMg}=4.21)$ el cual contrasta con los 11.28 reportados por Trejo (2005) específicamente para la UMA El Limón cuando realizó muestreos durante la época de lluvias. El promedio del índice de Shannon para las tres UMA's muestra una diversidad baja; en la UMA Ajuchitlan (2.39), El Limón (2.34) y en 
Cuadro 3. Distribución por categorías de las especies más dominantes de las UMA's en estudio.

\begin{tabular}{lccc}
\hline \multicolumn{1}{c}{ Categoría } & Ajuchitlan & UMA El Limón & Pitzotlan \\
\hline Especies con $>10 \%$ & 2 especies & 2 especies & 2 especies \\
de dominancia & Euphorbia & Ficus cotinifolia & Cordia curassavica \\
& Schlechtendalii & Lysiloma divaricata & Euphorbia \\
& Mimosa benthami & & schlechtendalii \\
Especies entre 1 y 10 & $29.31^{*}$ & $27.12^{*}$ & $34.15^{*}$ \\
$\%$ de dominancia & 12 especies & 19 especies & 17 especies \\
Especies con $<1 \%$ de & $60.67^{*}$ & $55.52^{*}$ & $65.83^{*}$ \\
dominancia & 26 especies & 33 especies & 19 especies \\
\hline
\end{tabular}

* Este porcentaje es la suma de la dominancia de las especies correspondientes a cada UMA.

Pitzotlan (1.90) ante una notable dominancia de Euphorbia schlechtendalii y Cordia curassavica. Sin embargo, los valores son bajos, comparados con otras SBC de México donde se presentan valores de 4.7 para la Chamela, Jalisco (Lott et al. 1987), 3.91 en Sayil, Yucatán (Trejo \& Dirzo 2002) y 3.63 en el ejido El Limón (Trejo 2005). La Uniformidad de Shannon fue similar en las tres UMA's, los valores fueron altos en las UMA's Ajuchitlan y El Limón (0.852 y 0.857, respectivamente) y no así para Pitzotlan. Los resultados anteriores concuerdan con el valor de 0.82 encontrados por Díaz et al. (2002) en una selva baja su perennifolia de Campeche y de 0.83 encontrados por Trejo (2005) en el ejido El Limón. El índice de Simpson es más sensible a los cambios en las abundancias de las especies comunes, por lo tanto, el nivel de dominancia es alto para las tres UMA's por algunas especies (Cuadro 3). El inverso de Simpson es alrededor de 50\% menor en el Limón (10.30) para éste estudio, comparado con lo encontrado (21.57) por Trejo (2005). De acuerdo con los valores obtenidos en el índice de Berger-Parker, la diversidad más alta se presentó la UMA Pitzotlan con 0.34 (Magurran 1989, Stilling 2002). Lo cual indica que las UMA's están dominadas por algunas especies (Cuadro 3), de esta manera se afecta la distribución de las mismas, los valores encontrado en las UMA's para este índice fueron valores altos comparados (0.20) con los encontrado por Gallardo et al. (2005) para una SBC en Nizanda, Oaxaca. La diferencia de los índices calculados en el presente estudio, comparado con otros estudios relacionados, probablemente se deba a los sitio de muestreo, la época del año en la que se realizó el muestreo, al tamaño de la muestra y a la densidad y dominancia que presenta la especie Euphorbia schlechtendalii en las UMA's del estudio. De acuerdo con Corral et al. (2002), los índices de Margalef y Shannon aumentan con el área de muestreo y los índices de Simpson y Menhinick disminuyen al hacerlo.

En la UMA El Limón se estimó mayor diversidad vegetal, esto tiene relevancia porque de acuerdo con Gallina et al. (1981) durante la época de sequía la diversidad vegetal en la dieta del venado cola blanca es mayor, resultado de la igualdad en el 
consumo de las diferentes formas biológicas de especies vegetales, mientras que en la época de lluvias la diversidad vegetal disminuye, porque incrementa la cantidad de herbáceas consumidas.

Como medio de información para orientar y garantizar la obtención de trofeos a los cazadores con permiso durante la época hábil del venado cola blanca, se eligieron los mejores transectos de acuerdo a las características del hábitat en este estudio y a la densidad de población estimada para los mismos transectos por Hernández (2008), en la UMA Ajuchitlan fueron Amate Amarillo, El Hoyo y Los Becerros; en la UMA El Limón fueron Cerro Prieto y Trancas y en la UMA Pitzotlan fueron Tepetates Blancos, Pueblo Viejo y Cerro Grande.

Los resultados de este estudio, indican que el hábitat del venado cola blanca en las UMA's de la Sierra de Huautla, Morelos, esta determinado principalmente por: a) disponibilidad de alimento; accesibilidad a la biomasa que forrajea; b) mayor diversidad y uniformidad de plantas preferidas por el venado; c) cobertura de protección y d) menor competencia con el ganado bovino. La presencia de agua fue indiferente, porque tanto en aquellos transectos donde se encontraban cuerpos de agua permanentes o no permanentes y en donde no los había, existió mayor o menor número de venados. Además que el hábitat del venado cola blanca determina todas las características de calidad del animal, así como un indicador del estado ecológico de las UMA's y a nivel de Reserva de la Biosfera. Entonces el proceso de concientización que han realizado los pobladores humanos de las UMA's para la conservación y aprovechamiento sustentable de los recursos naturales, principalmente el venado cola blanca y su hábitat, es un logro con grandes resultados a mediano y largo plazo.

AGRADECIMIENTOS. A los ejidatarios de las UMA's de la Sierra de Huautla, por todas las facilidades, tiempo, esfuerzo y amistad ofrecida durante el estudio realizado, así como por su hospitalidad otorgada a la Universidad Autónoma Chapingo durante los años de trabajo dentro de sus comunidades. Se agradece también a los estudiantes del Departamento de Agroecología de la misma universidad, por el apoyo ofrecido durante el estudio, al Departamento de Zootecnia y el Centro Regional Universitario del Anáhuac de la Universidad Autónoma Chapingo por el financiamiento otorgado.

\section{LITERATURA CITADA}

Arias, M.A., O. Dorado O. \& B. Maldonado. 2002. Biodiversidad e importancia de la Selva Baja Caducifolia: La Reserva de la Biosfera Sierra de Huautla. Biodiversitas, 7: 7-12.

Ávalos, H.O. 2007. Bombylidae (Insecta: Díptera) de Quilamula en el área de la Sierra de Huautla, Morelos, México. Acta Zoológica Mexicana (nueva serie), 23: 139-169.

Ayala, A.B.C. \& E.J. Quintero. 2003. Plan de manejo para venado cola blanca (Odocoileus virginianus) y guajolote silvestre (Meleagris gallopavo) en la UMA Los Romeros, Santiago Tulantepec, Hidalgo. Tesis de Ingeniería en Planeación y Manejo de los Recursos Naturales Renovables. Universidad Autónoma Chapingo. Chapingo, México.

Bello, G.J., S. Gallina \& M. Equihua. 2004. Distancias de desplazamiento del venado cola blanca y su relación con factores ambientales en el noroeste de México, pp. 146-151. In: Memorias del VI 
Congreso Internacional sobre Manejo de Fauna Silvestre en la Amazonia y Latinoamérica. Iquitos. Perú.

Castro, F.R. \& M.G. Bustos. 2003. Lagartijas de Morelos, México: distribución, hábitat y conservación. Acta Zoológica Mexicana (nueva serie), 88: 123-142.

Castro, F.R., G.G.G. Vergara, Z.M.G. Bustos \& A.W. Mena. 2006. Diversidad y distribución de anfibios en el estado de Morelos. Acta Zoológica Mexicana (nueva serie), 22: 103-117.

CONANP. 2005. Programa de conservación y manejo de la Reserva de la Biosfera Sierra de Huautla México. Comisión Nacional de Áreas Naturales Protegidas (CONANP). México.

Corona, Z.P. 2003. Bases biológicas para el aprovechamiento del venado cola blanca en el Ejido "El Limón de Cuauchichinola”, Municipio de Tepalcingo, Morelos. Tesis de Maestría en Ciencia. Manejo de Fauna silvestre. INECOL. Xalapa, Veracruz. México.

Corral, R.J., C.O.A. Aguirre, P.J. Jiménez \& C.J. de J. Nóvar. 2002. Muestreo de diversidad y observaciones ecológicas del estrato arbóreo del bosque mesófilo de montaña El Cielo, Tamaulipas, México. Revista Chapingo Serie Ciencias Forestales y del Ambiente, 8: 125-131.

Cox, W.G. 1976. Laboratory manual of general ecology. $3^{\text {a }}$ ed. Ed. WCB. Iowa. EUA.

Cruz, L.C. 2004. Densidad de población de venado cola blanca (Odocoileus virginianus) en el Ejido Pitzotlan, Tepalcingo Morelos. Documento de Titulación para la opción servicio universitario. Departamento de Suelos. Universidad Autónoma Chapingo. Chapingo, México.

Delfín-Alfonso, C. \& S. Gallina. 2007. Modelo de evaluación de hábitat para el venado cola blanca en un bosque tropical caducifolio en México. Capítulo 15, pp. 193-202. In: M. Zunino y A. Melic (Eds.). "Escarabajos, Diversidad y Conservación Biológica. Ensayos en homenaje a Gonzalo Halftter”. Monografías del 3er. Milenio Vol. 7. Sociedad Entomológica Aragonesa. España.

Díaz, G.J., O. Castillo \& García G. 2002. Distribución espacial y estructura arbórea de la selva baja subperennifolia en un ejido de la Reserva de la Biosfera de Calakmul, Campeche, México. Universidad y Ciencia, 38: 11-28.

Dorado, O., M.D. Arias, G. Alonso \& B. Maldonado. 2002. Educación ambiental para la biodiversidades el trópico seco, Reserva de la Biosfera Sierra de Huautla, Morelos, México. Tópicos en Educación Ambiental, 4: 23-33.

Duran, E., A.J. Meave, J.E. Lott \& G. Segura. 2006. Structure and tree diversity patterns at the landscapes level in a Mexican tropical deciduous forest. Boletín de la Sociedad Botánica de México, 79: 43-60.

Drawe, L.D. \& W.T. Box. 1968. Forage ratings for deer and cattle on the Welter wildlife refuge. Journal of Range Management, 47: 225-228.

Everitt, H.J. \& L.D. Drawe. 1974. Spring food habits of white tailed deer in the South Texas plains. Journal of Range Management, 27: 15-20.

Gallardo, C.J.A., A.J. Meave \& G.E.A. Peréz. 2005. Estructura, composición y diversidad de la selva baja caducifolia del Cerro Verde, Nizanda (Oaxaca), México. Boletín de la Sociedad Botánica de México, 76: 19-35.

Gallina, T.S.A. 1993. White-tailed deer and cattle diets at La Michilía, Durango, Mexico. Journal of Range Management, 46: 487-492.

Gallina, S., E. Maury \& V. Serrano. 1981. Food habits of White-tailed deer, pp. 133-148. In:. P. F. Ffolliot and S. Gallina. (Eds.). Deer biology, habitat requirements, and management in Western North America. Instituto de Ecologia, A. C. D. F. Mexico.

Gallina, S. 1998. Evaluación del hábitat para el venado, pp. 15-24. In: Primera Reunión Regional sobre venado cola blanca mexicano, Curso-Taller-Memorias. Puebla. México.

Gallina, S., J. Bello, C. Contreras \& C. Delfín-Alfonso. 2010. Daytime bedsite selection by the texan white-tailed deer in xerophyllous brushland, Northeastern, Mexico. Journal of Arid Environments, 74: $373-377$. 
García, E. 1981. Modificaciones al sistema de Köppen para adaptarlo a las condiciones de la República Mexicana. Ed. Larios, México.

González, B.G.T. 2005. Densidad de población del venado cola blanca (Odocoileus virginianus mexicanus), en la Sierra de Huautla, Morelos. Agencia de Desarrollo Local "Sierra de Huautla", Mor. Universidad Autónoma Chapingo. Chapingo. México.

González, B.G.T. 2006. Densidad de población del venado cola blanca (Odocoileus virginianus mexicanus), en la Sierra de Huautla, Morelos. Agencia de Desarrollo Local "Sierra de Huautla", Mor. Universidad Autónoma Chapingo. Chapingo. México.

González, B.G.T. \& L.A. López. 2008. Flora del pastizal tropical de la selva baja caducifolia. Tesis profesional. Universidad Autónoma Chapingo. Chapingo, México.

Gutiérrez, R.J. \& A.F. Squeo. 2004. Importancia de los arbustos en los ecosistemas semiáridos en Chile. Ecosistemas. Revista Cientifica y Técnica de Ecología y Medio Ambiente. 9(1).

Fulbright, T.E. \& A. Ortega. 2007. Ecología y manejo de venado cola blanca. Texas A\&M University Press, College Station.

Hernández, S.D.A. 2008. Venado cola blanca y su hábitat (Odocoileus virginianus mexicanus Z.), en Sierra de Huautla, Morelos. Tesis profesional. Universidad Autónoma Chapingo. Chapingo, México.

Lamprecht, H. 1990. Silvicultura en los Trópicos: Los ecosistemas forestales en los bosques tropicales y sus especies arbóreas-posibilidades y métodos para un aprovechamiento sostenido. G.T.Z. Eschborn. Alemania.

López-Téllez, M.C, C. Mandujano \& G. Yanés. 2007. Evaluación poblacional del venado cola blanca en un bosque tropical seco de la Mixteca Poblana. Acta Zoológica Mexicana (nueva serie), 23: $1-16$.

Lott, E., Bullock, H.S. \& A. Solís-Magallanes. 1987. Floristic diversity of upland and Arroyo forest Coastal Jalisco. Biotropica, 19: 228-235.

Lyons, K.R. \& F.T. Ginnett. 2001. Manejo integrado de hábitat para venado, codorniz y guajolote. Cooperativa de Texas. Extensión. Universidad Texas A\&M. Texas. EUA.

Magurran, E.A. 1989. Diversidad ecológica y su medición. Ed. Vedrá. Madrid, España.

Mandujano, S. 1994. Métodos para evaluar el hábitat del venado cola blanca en un bosque de coníferas. Pp. 283-297. In: Vaughan, C. y Rodríguez, M. A. (Eds). Ecología del venado cola blanca en México y Costa Rica. Organización de los Estados Americanos y Fundación de Vida Silvestre. San José, Costa Rica.

McMahan, A.C. \& W. Ramsey. 1965. Response of deer and livestock to controlled grazing in Central Texas. Journal of Range Management, 1: 1-7.

Montemayor, E., E.T. Fulbright, W.L. Brothers, J.B. Schat \& D. Cassels. 1991. Long term effects of rangeland disking on white tailed deer browse in south Texas. Journal of Range Management, 44: 246-248.

Muller-Dumbois, D. \& H. Ellenberg. 1974. Aims and methods of vegetation ecology. Ed. John Wiley and Sons New York. N. Y. EUA.

Mori, A.S., M.B. Boom, M.A. de Cervalino \& S.T. dos Santos. 1983. Ecological importance of Myrtaceae in an Eastern Brazilian Wet Forest. Biotropica, 15: 68-70.

Nogueda, A.C. 2007. Valor de nutritivo de la dieta del venado cola blanca (Odocoileus virginianus Z), en Sierra de Huautla, Morelos. Tesis profesional. Universidad Autónoma Chapingo. Chapingo, México.

Ortiz, M.T., S. Gallina, S.M. Briones \& G. González. 2005. Densidad de población y caracterización del hábitat del venado cola blanca (Odocoileus virginianus, Goldam y Kellog, 1940) en un bos- 
que templado de la Sierra Norte de Oaxaca, México. Acta Zoológica Mexicana (nueva serie), 21: $65-78$.

Ramírez, A.J.E. \& C.M.G. Ramírez. 2002. Avifauna de la región oriente de la Sierra Huautla, Morelos, México. Anales del Instituto de Biología. Serie Zoológica, 73: 91-111.

Ramírez, G.R., J.B. Quintanilla \& J. Aranda. 1997. White tailed deer food habits in Northeastern Mexico. Small Ruminant Research, 25: 141-146.

Rodríguez, V.B. \& J.B. Woolley. 2005. La fauna de la familia Encryrtidae (Hymenoptera: Cholcidoidea) en el bosque tropical caducifolio de la Sierra de Huautla, Morelos. México. Sociedad Mexicana de Entomología A. C. Folia Entomológica Mexicana, 44: 147-155.

Sánchez, H.C. \& A.M. de L. Romero. 1995. Mastofauna silvestre del área de la Reserva Sierra de Huautla (con énfasis en la región noroeste). Revista Mexicana de Mastozoología, 4: 124-125.

SMN. 1976-200. Servicio Meteorológico Nacional. Comisión Nacional del Agua. Normales Climatológicas. Estaciones: El Limón, Huautla y Tepalcingo. http://smn.cna.gob.mx/

Soto, W.I.A. 2000. Estrategias de comportamiento de las hembras del venado cola blanca tejano durante la época de crianza. Tesis de Maestría en Ciencia. Manejo de Fauna silvestre. INECOL. Xalapa, Veracruz. México.

Starker, L.A. 2000. Fauna Silvestre de México. Ed. PAX. México, D.F.

Stiling, P.D. 2002. Ecology Theories and applications. $5^{\mathrm{a}}$ ed. Ed. Prentice Hall. Nueva Jersey. EUA.

Trejo, I. 2005. Análisis de la diversidad de la selva baja caducifolia de México, pp. 111-122. In: Halffter, G.J.S., P. Koleff, \& A. Melic, (Eds). Sobre diversidad biológica: El significado de las Diversidades Alfa, Beta y Gamma. Ed. M3m Monografías Tercer Milenio. vol. 4. S.E.A., Zaragoza. España.

Trejo, I. \& R. Dirzo. 2002. Floristic diversity of Mexican seasonally dry tropical forest. Biodiversity and Conservation, 11: 2063-2084.

Trejo, V.R.I. 1998. Distribución y diversidad de selvas bajas de México: relaciones con el clima y el suelo. Tesis de Doctorado en Ciencias. Biología. Facultad de Ciencias. UNAM. México.

Trefethen, J.B. 1964. Wildlife management and conservation. D.C. Heath and Co. Boston. EUA.

Velázquez, A., A. Torres \& G. Bocco. 2003. Las enseñanzas de San Juan. Investigación participativa para el manejo integral de recursos naturales. INE-SEMARNAT. México.

Villarreal G.J.G. 2006. Venado cola blanca, manejo y aprovechamiento cinegético. $2^{\mathrm{a}}$ ed. Unión Ganadera Regional de Nuevo León, Fundación PRODUCE Nuevo León A.C. Confederación Nacional de Organizaciones Ganaderas. Monterrey, Nuevo León. México.

Villarreal, E.B.O.A., V.R. Guevara, M.I. Cortés, H.J. Hernández, G.F.J. Franco, C. Castillo \& H.T. Barrera. 2007. Alimentación del venado cola blanca mexicano (Odocoileus virginianus mexicanus Z.), en el sur de Puebla, México. Sociedad Española de Ovinotecnia y Caprinotecnia. Memoria: XXXII Jornadas Científicas y XI Jornadas Internacionales de Ovinotecnia y Caprinotecnía. 


\section{APÉNDICE 1}

Especies vegetales encontradas en las UMA's.

\begin{tabular}{|c|c|c|c|c|c|}
\hline Nombre científico & Nombre común & Familia & F.B. & & $\mathrm{P}$ \\
\hline Acacia angustissima (Mill.) Kuntze & & Fabaceae & Arbusto & $(\mathrm{Pe})(\mathrm{c})$ & 1 \\
\hline N.I. 1 & Aguacatillo & F.N.I. & Árbol & $(\mathrm{Pe})(\mathrm{c})$ & 1 \\
\hline Ficus goldmanii Standl & Amate prieto & Moraceae & Árbol & $(\mathrm{Pe})(\mathrm{c})$ & 1 \\
\hline $\begin{array}{l}\text { Thevetia thevetioides (H.B.K.) K. } \\
\text { Schum. }\end{array}$ & Ayoyote, yoyote & Apocinaceae & Árbol & $(\mathrm{Pe})(\mathrm{c})$ & 2 \\
\hline $\begin{array}{l}\text { Leucopremna mexicana (A.DC.) } \\
\text { Standl }\end{array}$ & Bonete & Caricaceae & Árbol & $(\mathrm{Pe})(\mathrm{c})$ & 2 \\
\hline Haematoxylon brasiletto Karst. & Brasil & Fabaceae & Árbol & $(\mathrm{Pe})(\mathrm{c})$ & 1 \\
\hline Acalypha langiana Müell. & Cancerina & Euphorbiaceae & Arbusto & $(\mathrm{Pe})(\mathrm{c})$ & 1 \\
\hline Vitex pyramidata B.L. Robinson & $\begin{array}{l}\text { Canelillo o } \\
\text { querende }\end{array}$ & Verbenaceae & Árbol & $(\mathrm{Pe})(\mathrm{c})$ & 2 \\
\hline Dalbergia congestiflora Pittier & Carpinceran & Fabaceae & Árbol & $(\mathrm{Pe})(\mathrm{c})$ & 2 \\
\hline Ipomoea wolcottiana Rose & $\begin{array}{l}\text { Casahuate } \\
\text { amarillo }\end{array}$ & Convolvulaceae & Árbol & $(\mathrm{Pe})(\mathrm{c})$ & 1 \\
\hline Ipomoea murocoides Roem et Schult & Casahuate prieto & Convolvulaceae & Árbol & $(\mathrm{Pe})(\mathrm{c})$ & 2 \\
\hline $\begin{array}{l}\text { Ipomoea arborescens (Humb. et } \\
\text { Bonpl.) Don. }\end{array}$ & $\begin{array}{l}\text { Casahuate } \\
\text { blanco }\end{array}$ & Convolvulaceae & Árbol & $(\mathrm{Pe})(\mathrm{c})$ & 1 \\
\hline Stemmadenia bella Miers. & Chicliyo & Apocinaceae & Arbusto & $(\mathrm{Pe})(\mathrm{c})$ & 2 \\
\hline N.I. 6 & Chipil & Fabaceae & Arbusto & $(\mathrm{Pe})(\mathrm{c})$ & 1 \\
\hline Gouania lupuloides (L.) Urb. & Chupamito & Rhamnaceae & Arbusto & $(\mathrm{Pe})(\mathrm{c})$ & 1 \\
\hline Spondias purpurea L. & Ciruelo & Anacardiaceae & Árbol & $(\mathrm{Pe})(\mathrm{c})$ & 1 \\
\hline N.I. 5 & Cola de zorra & Acanthaceae & Arbusto & $(\mathrm{Pe})(\mathrm{c})$ & 2 \\
\hline $\begin{array}{l}\text { Bursera bipinnata (Sessé \& Moc) } \\
\text { Engl. }\end{array}$ & Copal chino & Burseraceae & Árbol & $(\mathrm{Pe})(\mathrm{c})$ & 1 \\
\hline Bursera glabrifolia (H.B.K) Engl. & Copal manso & Burseraceae & Árbol & $(\mathrm{Pe})(\mathrm{c})$ & 1 \\
\hline $\begin{array}{l}\text { Bursera copallifera (Sessé \& Moc. Ex } \\
\text { DC.) Engl. }\end{array}$ & Copal ancho & Burseraceae & Árbol & $(\mathrm{Pe})(\mathrm{c})$ & 1 \\
\hline Juliana adstringens Schlencht & Cuachalalate & Julianaceae & Árbol & $(\mathrm{Pe})(\mathrm{c})$ & 2 \\
\hline Guazuma ulmifolia Lam. & Cuaulote & Stercuiliaceae & Árbol & $(\mathrm{Pe})(\mathrm{c})$ & 1 \\
\hline Bursera lacifolia (Schl. Engl) & Cuajiote blanco & Burseraceae & Árbol & $(\mathrm{Pe})(\mathrm{c})$ & 1 \\
\hline Bursera longipes (Rose) Standl. & Cuajiote rojo & Burseraceae & Árbol & $(\mathrm{Pe})(\mathrm{c})$ & 1 \\
\hline Crescentia cujete L. & Cuatecomate & Bignoniaceae & Árbol & $(\mathrm{Pe})(\mathrm{c})$ & 1 \\
\hline Heliocarpus reticulatus Rose & Cuaulahuatl & Tiliaceae & Árbol & $(\mathrm{Pe})(\mathrm{c})$ & 2 \\
\hline Acacia acatlensis Benth & Cubata blanca & Fabaceae & Árbol & $(\mathrm{Pe})(\mathrm{c})$ & 1 \\
\hline
\end{tabular}


Especies vegetales encontradas en las UMA's (Continúa).

\begin{tabular}{|c|c|c|c|c|c|}
\hline Nombre científico & Nombre común & Familia & F.B. & & $\mathrm{P}$ \\
\hline $\begin{array}{l}\text { Acacia cochliacantha Humb. \& Bonpl } \\
\text { ex Willd }\end{array}$ & Cubata prieta & Fabaceae & Árbol & $(\mathrm{Pe})(\mathrm{c})$ & 1 \\
\hline Montanoa speciosa (DC) Shultz Bip. & Cuilote & Asteraceae & Arbusto & $(\mathrm{Pe})(\mathrm{c})$ & 1 \\
\hline Quercus glaucoides Mart \& Gal & Encino blanco & Fagaceae & Árbol & $(\mathrm{Pe})(\mathrm{c})$ & 1 \\
\hline $\begin{array}{l}\text { Acacia pennatula (Schlecht. \& Cham) } \\
\text { Benth. }\end{array}$ & Espino blanco & Fabaceae & Árbol & $(\mathrm{Pe})(\mathrm{c})$ & 1 \\
\hline $\begin{array}{l}\text { Myrtillocactus geometrizans (Martius) } \\
\text { Consele }\end{array}$ & Garambullo & Cactaceae & Arbusto & $(\mathrm{Pe})(\mathrm{c})$ & 1 \\
\hline Randia watsoni Robinson & Grangel blanco & Rubiaceae & Arbusto & $(\mathrm{Pe})(\mathrm{c})$ & 1 \\
\hline Leucaena collinsii Britton \& Rose & Guaje colorado & Fabaceae & Árbol & $(\mathrm{Pe})(\mathrm{c})$ & 1 \\
\hline Malpigia mexicana Jussie & Guajocote & Malpighiaceae & Árbol & $(\mathrm{Pe})(\mathrm{c})$ & 1 \\
\hline N.I. 2 & Guaspelon & Fabaceae & Árbol & $(\mathrm{Pe})(\mathrm{c})$ & 1 \\
\hline Ruprechtia fusca Fern & $\begin{array}{l}\text { Guayabillo f/ } \\
\text { alas }\end{array}$ & Polygonaceae & Árbol & $(\mathrm{Pe})(\mathrm{c})$ & 1 \\
\hline Pterocarpus acapulcensis Rose & $\begin{array}{l}\text { Guayacán } \\
\text { amarillo }\end{array}$ & Fabaceae & Árbol & $(\mathrm{Pe})(\mathrm{c})$ & 1 \\
\hline $\begin{array}{l}\text { Conzattia multiflora (B. L.Rob.) } \\
\text { Standl. }\end{array}$ & $\begin{array}{l}\text { Guayacán } \\
\text { blanco }\end{array}$ & Fabaceae & Árbol & $(\mathrm{Pe})(\mathrm{c})$ & 1 \\
\hline Gyrocarpus americanus Jacq. & Hediondillo & Hernandiaceae & Árbol & $(\mathrm{Pe})(\mathrm{c})$ & 2 \\
\hline Bunchosia canescens D.C. & $\begin{array}{l}\text { Hierba del } \\
\text { coyote }\end{array}$ & Malpighiaceae & Arbusto & $(\mathrm{Pe})(\mathrm{c})$ & 2 \\
\hline N.I. 3 & Huachipil & Fabaceae & Árbol & $(\mathrm{Pe})(\mathrm{c})$ & 2 \\
\hline Acacia farnesiana (L.) Willd & Huisache & Fabaceae & Árbol & $(\mathrm{Pe})(\mathrm{c})$ & 1 \\
\hline Euphorbia schlechtendalii Boiss. & Ixtumeca & Euphorbiaceae & Arbusto & $(\mathrm{Pe})(\mathrm{c})$ & 1 \\
\hline Agave angustifolia Haw & Maguey & Agavaceae & Arbusto & $(\mathrm{Pe})$ & 1 \\
\hline Cordia curassavica (Jacq.) R. \& S. & $\begin{array}{l}\text { Manzana } \\
\text { alargada roja }\end{array}$ & Boraginaceae & Arbusto & $(\mathrm{Pe})(\mathrm{c})$ & 2 \\
\hline Cordia inermis (Mill.) I. M. Jonnston & $\begin{array}{l}\text { Manzana } \\
\text { redonda }\end{array}$ & Boraginaceae & Arbusto & $(\mathrm{Pe})(\mathrm{c})$ & 2 \\
\hline Lippia berlandieri Schauer in D.C. & $\begin{array}{l}\text { Manzanita } \\
\text { morada }\end{array}$ & Verbenaceae & Arbusto & $(\mathrm{Pe})(\mathrm{c})$ & 1 \\
\hline $\begin{array}{l}\text { Mimosa albida Humb. \& Bonpl. ex } \\
\text { Wild }\end{array}$ & & Fabaceae & Arbusto & $(\mathrm{Pe})(\mathrm{c})$ & 1 \\
\hline Opuntia lubrica Griffiths & Nopal & Cactaceae & Arbusto & $(\mathrm{Pe})$ & 1 \\
\hline Pachycereus grandis Rose & Órgano & Cactaceae & Arbusto & $(\mathrm{Pe})$ & 1 \\
\hline N.I. 4 & Palillo & F.N.I. & Arbusto & $(\mathrm{Pe})(\mathrm{c})$ & 2 \\
\hline Eysenhardtia polystachya (Ort) Sarg & Palo dulce & Fabaceae & Árbol & $(\mathrm{Pe})(\mathrm{c})$ & 1 \\
\hline
\end{tabular}


Especies vegetales encontradas en las UMA's (Continúa).

\begin{tabular}{|c|c|c|c|c|c|}
\hline Nombre científico & Nombre común & Familia & F.B. & & $\mathrm{P}$ \\
\hline Bursera grandifolia (Schl.) Engl. & Palo mulato & Burseraceae & Árbol & $(\mathrm{Pe})(\mathrm{c})$ & 1 \\
\hline Arctostaphylos polifolia H.B.K. & $\begin{array}{l}\text { Palo prieto, } \\
\text { pingüica }\end{array}$ & Ericaceae & Árbol & $(\mathrm{Pe})(\mathrm{c})$ & 1 \\
\hline Senna skinneri Benth & Paráca & Fabaceae & Árbol & $(\mathrm{Pe})(\mathrm{c})$ & 1 \\
\hline $\begin{array}{l}\text { Lemaireocereus stellatus (Pfeiffer) } \\
\text { Briton and Rose }\end{array}$ & Pitaya & Cactaceae & Árbol & $(\mathrm{Pe})(\mathrm{c})$ & 1 \\
\hline Ceiba parvifolia Rose & Pochote de secas & Bombacaceae & Árbol & $(\mathrm{Pe})(\mathrm{c})$ & 1 \\
\hline Lonchocarpus eriophyllus Benth & Quebracha & Fabaceae & Árbol & $(\mathrm{Pe})(\mathrm{c})$ & 1 \\
\hline Amaranthus hybridus L. & Quelite & Amaranthaceae & Arbusto & $(\mathrm{Pe})(\mathrm{c})$ & 1 \\
\hline Waltheria americana $\mathrm{L}$. & $\begin{array}{l}\text { Tapacola, } \\
\text { manrubio }\end{array}$ & Stercuiliaceae & Arbusto & $(\mathrm{Pe})(\mathrm{c})$ & 2 \\
\hline Trichilia hirta L. & Tapaqueso & Meliaceae & Árbol & $(\mathrm{Pe})(\mathrm{c})$ & 2 \\
\hline Mimosa benthami Macbride & Tecolhuixtle & Fabaceae & Árbol & $(\mathrm{Pe})(\mathrm{c})$ & 1 \\
\hline Lysiloma acapulcensis (Kunth) Benth & Tepeguaje & Fabaceae & Árbol & $(\mathrm{Pe})(\mathrm{c})$ & 1 \\
\hline Coursetia glandulosa A. Gray & Tepexoco & Fabaceae & Árbol & $(\mathrm{Pe})(\mathrm{c})$ & 2 \\
\hline Bursera bicolor (Willd.) Engl. & Ticumaca & Burseraceae & Árbol & $(\mathrm{Pe})(\mathrm{c})$ & 1 \\
\hline Lysiloma divaricata (Jacq.) McBride & Tlahuitol & Fabaceae & Árbol & $(\mathrm{Pe})(\mathrm{c})$ & 1 \\
\hline Ficus cotinifolia H.B.K. & Tlaligo & Moraceae & Árbol & $(\mathrm{Pe})(\mathrm{c})$ & 1 \\
\hline Mimosa polyantha Benth & Uña de gato & Fabaceae & Arbusto & $(\mathrm{Pe})(\mathrm{c})$ & 1 \\
\hline Cassia biflora L. & Varadura & Fabaceae & Arbusto & $(\mathrm{Pe})(\mathrm{c})$ & 1 \\
\hline Sapium macrocarpum Muell. Arg. & $\begin{array}{l}\text { Veneno, } \\
\text { venenillo }\end{array}$ & Euphorbiaceae & Árbol & $(\mathrm{Pe})(\mathrm{c})$ & 2 \\
\hline Celtis caudata Planch. & Zazanaque & Ulmaceae & Árbol & $(\mathrm{Pe})(\mathrm{c})$ & 2 \\
\hline
\end{tabular}

F.B. (Forma biológica), N. I. (Especie no identificada), F.N.I (Familia no identificada), (Pe) Perenne; (c) (caducifolio), (P) Preferencia 1: Consumida; 2: no consumida, por el venado cola blanca en la Sierra de Huautla, Morelos, México. 